\title{
Artroplastia total de muñeca. Resultados funcionales, complicaciones y factores predictivos de la evolución
}

\author{
A. Aragó, B. Morales Moreno, M. García López, J. M. Valmaña de la Sotilla, \\ L. Jiménez Alcázar, M. Á. Martínez Calvo \\ Servicio de Cirugía Ortopédica y Traumatología del Hospital Universitario Príncipe de Asturias. Alcalá de Henares. España.
}

Resumen: Objetivo: El objetivo de este estudio es presentar los resultados a corto plazo de la artroplastia total de muñeca y estudiar los factores que determinan su evolución.

Material y método: Se han revisado retrospectivamente 15 pacientes intervenidos mediante prótesis total de muñeca Tipo Universal ${ }^{\circledR}$ (KMI, Carlsbad, California). Se han estudiado los factores epidemiológicos, las radiografías, la movilidad y la fuerza prequirúrgica y postquirúrgica. Se han evaluado a los pacientes mediante el cuestionario DASH y la escala visual analógica, EVA. Se han utilizado diversos test estadísticos para el estudio de la predicción de la evolución. Se han descrito las complicaciones y las reintervenciones.

Resultados: Los pacientes han presentado una disminución del dolor estadísticamente significativa. No hemos obtenido diferencias estadísticamente significativas de ninguno de los valores para prever la evolución. Seis de los casos requirieron ser reintervenidos debido a complicaciones como pinzamiento, "impingement" cubital, neuropatía del nervio cubital y luxación del primer compartimento extensor.

Conclusiones: La artroplastia total de muñeca es una alternativa en el tratamiento de la artrosis radiocarpiana avanzada en pacientes que quieren preservar cierta movilidad en la muñeca. Las complicaciones han surgido debido a que se trata de una exigente técnica quirúrgica, y disminuyen con la curva de aprendizaje. No se han encontrado factores predictivos de su evolución.

Palabras claves: Artroplastia de muñeca, artrosis radiocarpiana, Kienböck.

\section{Total wrist arthroplasty. Functional outcomes, complications and predictors of evolution}

\begin{abstract}
Objective: The purpose of this study was to present the short-term clinical results of total wrist arthroplasty and to explore possible factors determining the outcome.

Methods: We retrospectively reviewed 15 patients with a total wrist arthroplasty Universal ${ }^{\circledR}$ type implanted (KMI, Carlsbad, California). We studied epidemiologic factors, radiographs, wrist motion and grip strength preoperatively and postoperatively. We administered the visual analog pain scale and Disabilities of the arm, shoulder and hand questionnaire. We used some statistical tests to study the prediction of the evolution. We described the complications and the reinterventions. Results: The pain of the patients had a significant improvement. We didn't find any differences in any of the factors to predict the evolution. Six cases required reinterventions because of some complications such dislocations, ulnar impingement, ulnar neuropathy and volar subluxation of the tendons of the first dorsal compartment.

Conclusions: Total wrist arthroplasty is a good alternative in the treatment of advanced radiocarpal osteoarthritis in patients who want to preserve some wrist mobility. We have not found any factors to predict the evolution of this surgery.
\end{abstract}

Key words: Arthroplasty, total wrist replacement, radiocarpal osteoarthritis, Kienböck.

En un principio las indicaciones de la Artroplastia Total de Muñeca (ATM) estaban limitadas a estadios avanzados de artritis reumatoide, en pacientes con baja demanda funcional'. La complejidad de la articulación de la muñeca, los tratamientos alternativos existentes y una tasa elevada de complicaciones (hasta de un 75\%) no ha permitido que esta intervención ganase popularidad entre cirujanos y pacientes.

Recientemente, los avances en el diseño de las prótesis mejorando la cinemática y la fijación de los implan- tes, han ampliado las indicaciones de la misma, incluyendo estadios avanzados de artrosis primaria, de enfermedad de Kienböck y de artropatía degenerativa postraumática ${ }^{2-3-4}$. Su ventaja principal sobre la artro-
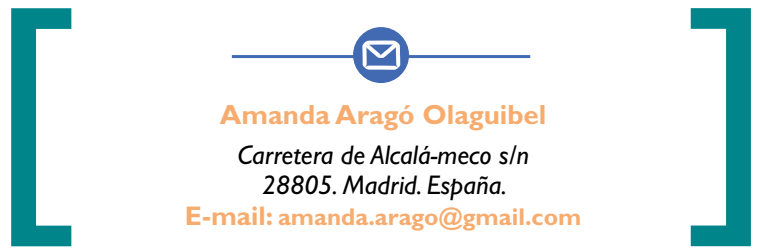
desis de muñeca radica en preservar cierta movilidad a cambio de una limitación en la fuerza.

El objetivo de este estudio es realizar un análisis descriptivo de los resultados clínicos de los pacientes intervenidos mediante ATM en la Unidad de Mano del Hospital Universitario Príncipe de Asturias a corto plazo y estudiar si existen factores que determinen la evolución de estos pacientes.

\section{MATERIALY MÉTODO}

\section{Pacientes}

Se realizó un estudio descriptivo retrospectivo en 15 pacientes que fueron intervenidos por el equipo de cirujanos de la Unidad de Mano del Hospital Universitario Príncipe de Asturias entre los años 2006 y 2011 implantándoles una prótesis total de muñeca tipo Universal $2^{\circledR}$ (KMI, Carlsbad, California). La artroplastia se indicó en pacientes con dolores radiocarpianos de diferentes etiologías, siempre que estuvieran afectadas las articulaciones radio-lunar y semilunar-grande, y que los pacientes estuvieran interesados en mantener unos grados de movilidad en la muñeca.

Se estudiaron sus datos epidemiológicos: el sexo, la edad, el lado dominante, su patología de base y sus intervenciones quirúrgicas previas en esa muñeca.

\section{Datos epidemiológicos}

La edad media en el momento de la intervención fue de 57,2 años, con un rango de 38-70 años. En cuanto al sexo, $60 \%$ fueron varones y $40 \%$ mujeres. El $66 \%$ de las intervenciones se realizó en la muñeca derecha dominante. Más de la mitad de los pacientes eran trabajadores manuales. El $46 \%$ de los pacientes habían sido intervenidos previamente de esa muñeca, siendo el número medio de intervenciones de 1,3 con un rango de |-7. El seguimiento medio fue de $4 \mid$ meses con un rango de $12-72$ meses.

En 7 de los casos la patología de base fue no traumática: 3 pacientes presentaban una enfermedad de Kienböck, I paciente una artropatía inflamatoria psoriásica y 3 pacientes una artrosis degenerativa de muñeca.

Por otro lado, 8 pacientes presentaron un antecedente traumático, representando el 53\% de los pacientes:
I caso de fractura de radio distal, 4 casos de muñeca SNAC y 3 de muñeca SLAC.

\section{Técnica quirúrgica}

En todos los casos, se intervino al paciente en decúbito supino, sobre mesa de mano, bajo anestesia locorregional y bajo isquemia con expresión de la extremidad. Se realizó un abordaje dorsal de la muñeca mediante incisión longitudinal. Se implantaron los 3 componentes de la prótesis siguiendo los pasos de la guía técnica, mediante las guías de osteotomía del radio y del carpo. No se cementaron los componentes. Se comprobó la adecuada colocación de los implantes y la estabilidad bajo radioscopia. Se asoció una exéresis de la cabeza del cúbito en 10 casos. Se realizó un cierre riguroso de la cápsula mediante reanclaje con arpones y reconstrucción del retináculo extensor.

\section{Protocolo postoperatorio}

Los pacientes mantuvieron una inmovilización mediante férula antebraquial entre 4 y 8 semanas, comenzando la rehabilitación en la tercera o cuarta semana postquirúrgica.

\section{Evaluación}

Los resultados se midieron, de forma pre y postquirúrgica, valorando el dolor con la Escala Visual Analógica EVA (0-10), siendo 0 la ausencia de dolor; la movilidad con un goniómetro (flexo-extensión, pronosupinación, desviación radial y desviación cubital) y la fuerza de prensión mediante un dinamómetro de Jamar. A todos los pacientes se les realizaron radiografías de control antero-posterior y lateral de muñeca, buscando la presencia de líneas de radiolucencia alrededor de los componentes protésicos.

Se valoraron las actividades de la vida diaria con el cuestionario DASH (Disabilities of the Arm, Shoulder, and Hand) validado en idioma Castellano ${ }^{5}$ únicamente de forma post-quirúrgica.

Para determinar si trabajábamos con variables normales o no, se utilizó el test de Shapiro-Wilks. Como test estadístico se utilizó la Prueba $U$ de Mann-Whitney, un test no paramétrico para variables independientes en una muestra pequeña. Se estudió la relación entre los factores epidemiológicos de los pacientes respecto a los resultados obtenidos de movilidad, fuerza, dolor y del cuestionario DASH.

Por último, se recogieron las complicaciones acontecidas y las reintervenciones realizadas. 


\section{RESULTADOS}

En cuanto a la movilidad, los pacientes pasaron de un rango medio de flexo-extensión prequirúrgica de $79^{\circ}$ a $68^{\circ}$ de movilidad postquirúrgica; de una desviación radial media prequirúrgica de $7,8^{\circ}$ a $4^{\circ}$ postquirúrgica; y de una desviación cubital media prequirúrgica de $25,5^{\circ}$ a $23^{\circ}$ postquirúrgica.

La disminución del dolor fue estadísticamente significativa según el test de los rangos de Wilcoxon, pasando de una puntuación media prequirúrgica de 8,4 a una puntuación media postquirúrgica de 3,73.

El porcentaje medio de fuerza respecto al miembro contralateral fue del $28 \%$.

La puntuación media del DASH fue de 40 sobre 100 con un rango de 10 a 83 .

En las radiografías no se observaron signos de aflojamiento protésico \& Figura I.
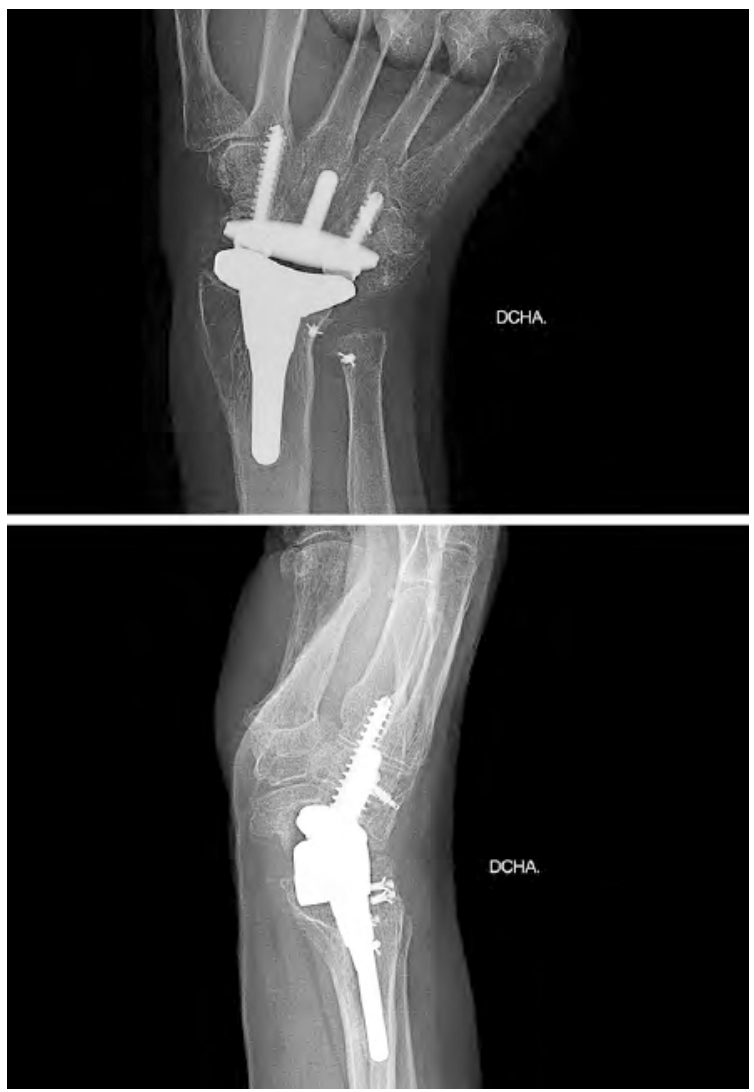

Figura ॥. Radiografías antero-posterior y lateral de prótesis total de muñeca. Seguimiento tras 3 años de evolución sin mostrar signos de aflojamiento.
No encontramos relación estadísticamente significativa, según la prueba de $U$ de Mann-Whitney, entre ser mayor o menor de 60 años, ser hombre o mujer, tener de base una patología traumática o no, haber padecido una enfermedad de Kienböck o no, haber sido intervenido previamente de esa muñeca o no, haber completado la cirugía con una resección de la cabeza cubital tipo Darrach o no, con la evolución de los pacientes, es decir, con el rango de movimiento, el dolor, el DASH o el porcentaje de fuerza postquirúrgicos.

\section{Complicaciones y reintervenciones}

Seis de los pacientes presentaron complicaciones mayores, entendiendo por éstas, aquéllas que necesitaron una reintervención quirúrgica, obteniendo una tasa de complicaciones mayores del $40 \%$.

Dentro de las complicaciones mayores podemos distinguir: tres fracasos de prótesis, en dos casos por luxación en el postoperatorio inmediato (2) Figura 2 y en un caso por secuela de dolor persistente, que se rescataron mediante artrodesis; y otras complicaciones asociadas entre las que se encuentran tres casos de pinzamiento cubital, un caso de neuropatía del nervio cubital y un caso de luxación del primer compartimento extensor que precisaron de osteotomía de Darrach, de liberación en el canal de Guyón y de recentrado del primer compartimento, respectivamente.

Como complicaciones menores, los pacientes sufrieron tendinitis de De Quervain y de los extensores, neuropatías del nervio mediano y del nervio cubital, que fueron temporales y se resolvieron con tratamiento conservador.

\section{DISCUSIÓN}

La artroplastia total de muñeca no consigue obtener tan buenos resultados como la artroplastia de rodilla o cadera pero poco a poco va haciéndose camino y mejorando la calidad de vida de los pacientes, pese a una tasa aún demasiado alta de complicaciones ${ }^{6}$. El elevado número de diferentes diseños protésicos indica que aún no se ha encontrado el implante perfecto para la anatomía y biomecánica de la muñeca'. Se necesita mayor variedad de tamaños en los componentes y mayor precisión en las guías de corte para conseguir resultados más reproducibles. 


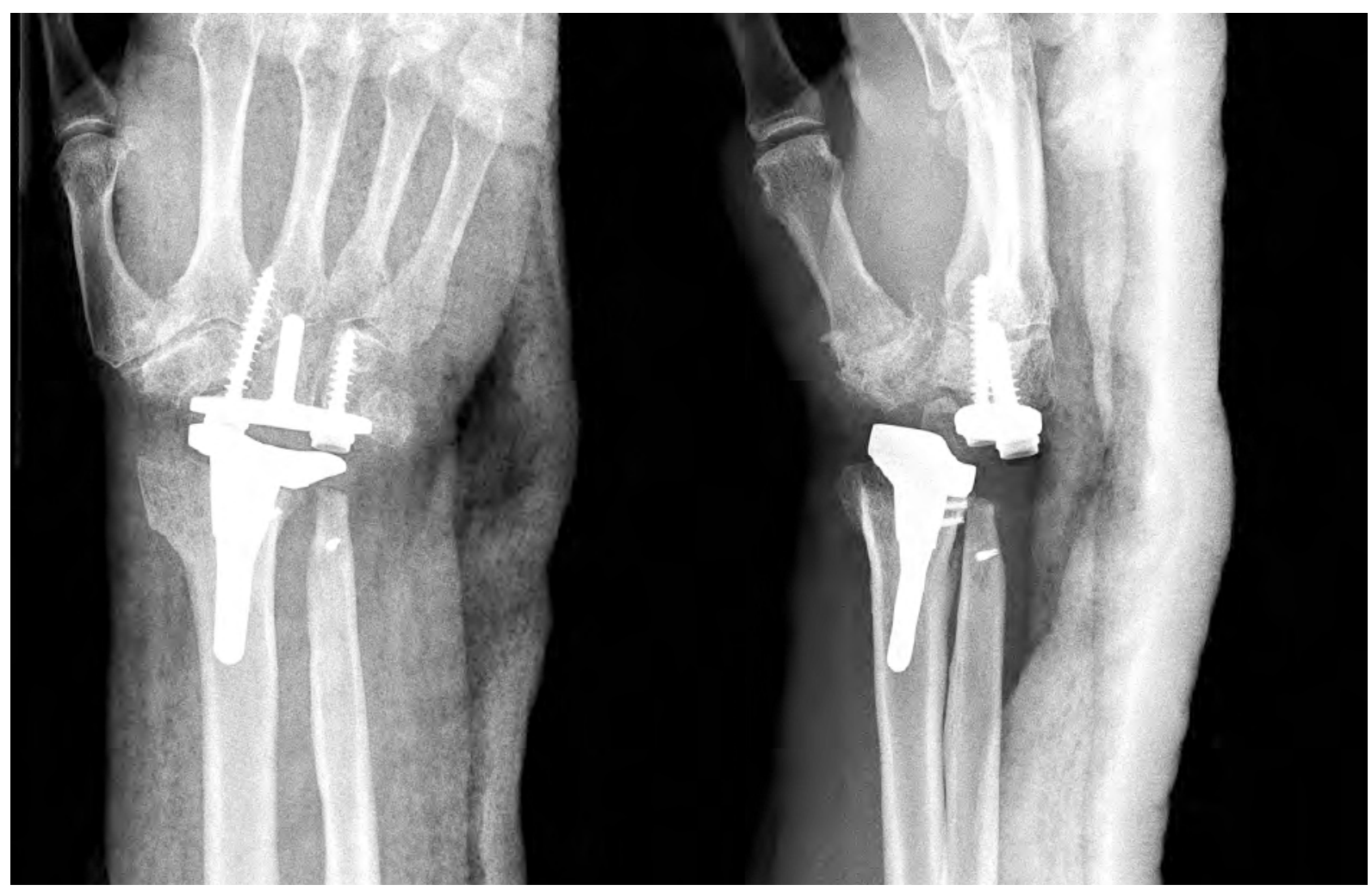

Figura 2. Radiografías antero-posterior y lateral de prótesis total de muñeca. Luxación de prótesis de muñeca en el postoperatorio inmediato.

La artrodesis de muñeca es un procedimiento con unos resultados predecibles que consigue una disminución del dolor y mantenimiento de fuerza a cambio de una pérdida completa de la movilidad, excepto de prono-supinación ${ }^{7-8-9}$. Sin embargo, la preservación de cierta movilidad, aunque sea limitada, puede proporcionar importantes beneficios en la vida de los pacientes $^{7-10}$. Algunos autores defienden que una muñeca para ser funcional solo requiere una movilidad de $30^{\circ}$ de extensión, $5^{\circ}$ de flexión, $10^{\circ}$ de desviación radial y $15^{\circ}$ de desviación cubital ${ }^{10}$, movilidad conseguida tras la implantación de la prótesis en nuestra serie. La ventaja de la artroplastia es que, en caso de fracaso, su reconversión a artrodesis de muñeca es posible y relativamente sencilla ${ }^{12-13}$.

La disminución o ausencia del dolor ha sido menor en nuestro hospital que la hallada en la bibliografía (2) Tabla I. Nuestra tasa de complicaciones mayores, $40 \%$, ha sido mayor que la de la mayoría de estudios ${ }^{12}$ (1) Tabla 2

Una colocación del componente radial excesivamente hacia cubital debido, en parte, a encontrarnos en el debut de la curva de aprendizaje $y$, en parte, a una mala traducción de la técnica quirúrgica aportada por la casa comercial, resultó en complicaciones en el postoperatorio inmediato en los primeros pacientes (1) Figura 3.

La no cementación de los implantes, debido a que nuestra serie estaba formada por adultos con buena calidad ósea, no ha influido en nuestros resultados pues no hemos tenido aflojamientos durante el seguimiento.

La decisión inicial de no resecar la cabeza del cúbito, debido a la ausencia de clínica en la articulación radiocubital distal de nuestros pacientes, en una prótesis diseñada en sus orígenes para pacientes con artropatía reumatoide con afectación de la articulación radiocubital distal, ha favorecido la aparición de impingement cubital, obligándonos a realizar posteriormente una exéresis de la cabeza del cúbito.

Una de las principales desventajas de este estudio es su carácter retrospectivo y la falta de datos preoperatorios para realizar comparaciones estadísticas. Se trata de un estudio con una muestra pequeña, a corto 


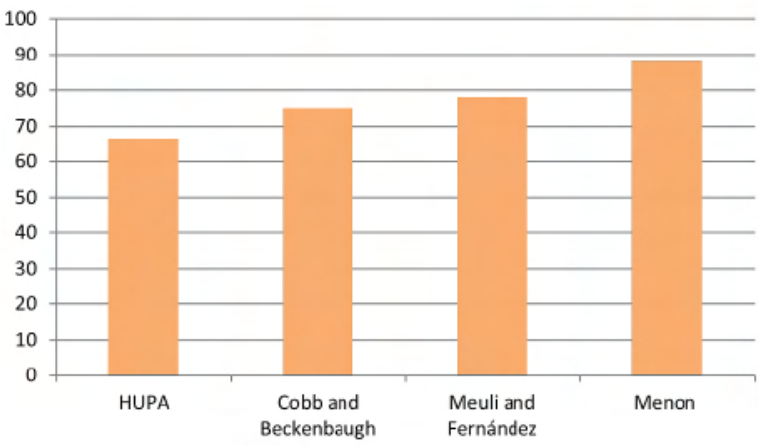

Tabla I. Porcentaje de pacientes con EVA postquirúrgico entre 0-3 en nuestro hospital en comparación con otros autores. HUPA: Hospital Universitario Príncipe de Asturias..

plazo y que solo nos da una evidencia exploratoria, que no conclusiva.

\section{CONCLUSIÓN}

Como conclusión, se puede decir que la artroplastia total de muñeca es una alternativa en el tratamiento de la artrosis radiocarpiana avanzada. El margen de error es muy pequeño ante mínimas variaciones en la orientación por lo que se trata de una cirugía que requiere una técnica minuciosa con osteotomías precisas, un perfecto balance de las partes blandas y un cierre meticuloso de la cápsula para obtener buenos

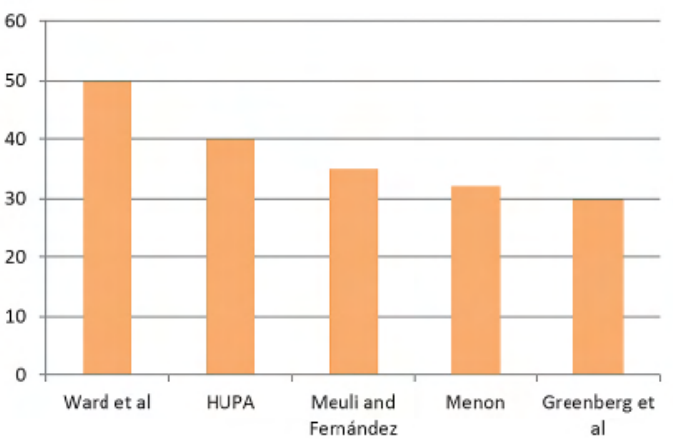

Tabla. II. Porcentaje de complicaciones en nuestro hospital respecto a otros autores. HUPA: Hospital Universitario Príncipe de Asturias.

resultados. Por supuesto, una buena selección de los pacientes es necesaria, descartando los pacientes con alta demanda funcional. Es necesario el control por radioscopia para una correcta colocación y orientación de los implantes. Se aconseja la realización de una resección de la cabeza cubital tipo Darrach asociado a la implantación de la prótesis.

Cuando se realiza una buena técnica en los pacientes apropiados la artroplastia de muñeca puede beneficiar al paciente con buenos resultados funcionales ${ }^{14}$.

Podemos afirmar que existe una diferencia estadísticamente significativa en la disminución del dolor. Pese
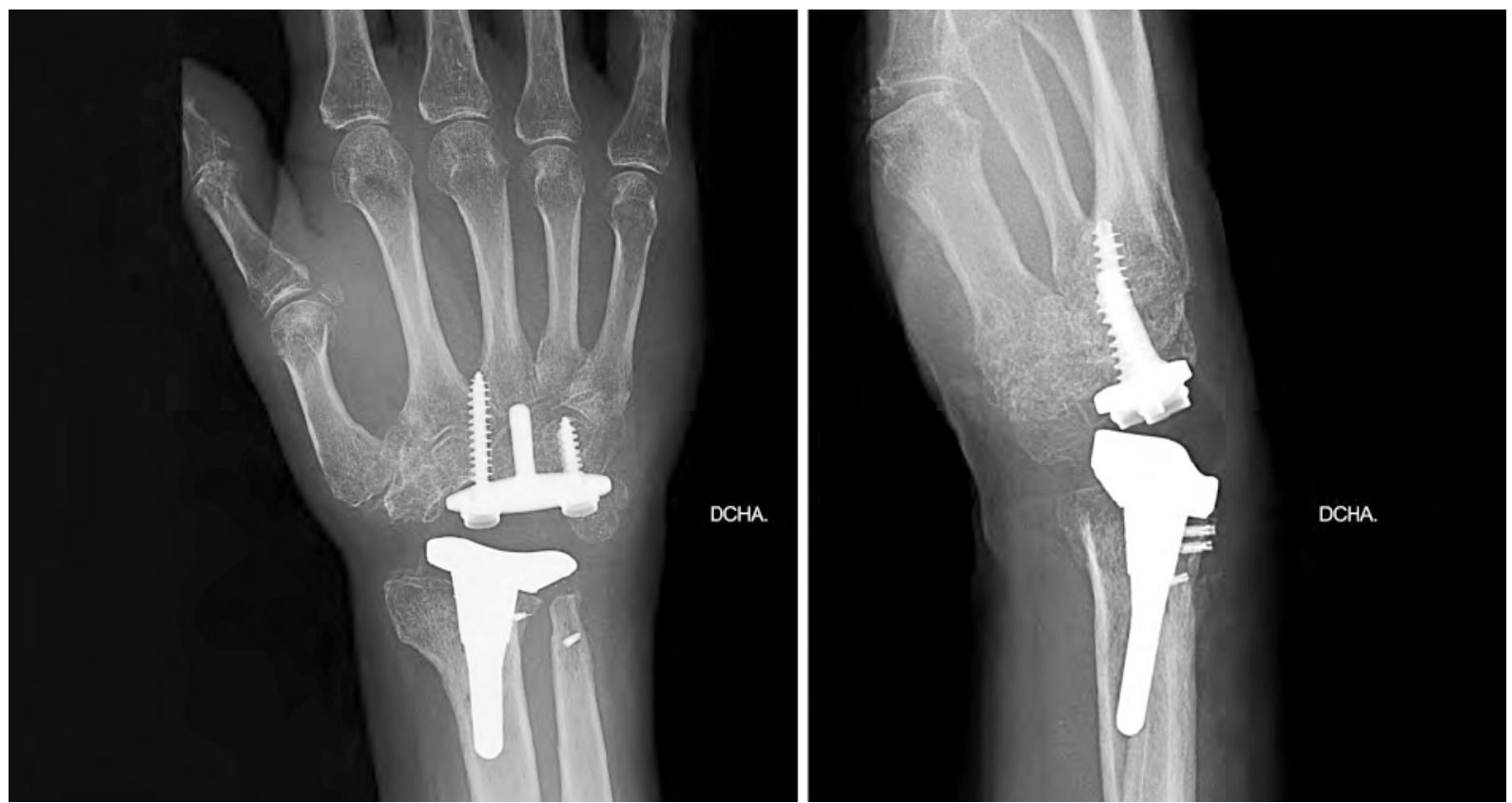

Figura 3. Radiografías antero-posterior y lateral de prótesis total de muñeca. Componente radial y tornillo carpiano radial excesivamente cubitalizados. 
a que los resultados muestran un déficit de fuerza, los pacientes encuentran aceptable su movilidad para las actividades de la vida diaria. No hemos encontrado ningún valor con diferencias estadísticamente significativas para prever una mejor o peor evolución de los pacientes que nos ayude a puntualizar mejor las indicaciones para este tipo de intervención.

La alta tasa de complicaciones parece estar relacionada con la complejidad de la articulación de la muñeca, una exigente técnica quirúrgica mejorada con la curva de aprendizaje y el diseño del implante.

Pensamos que se necesita una formación específica previa para la implantación de este tipo de prótesis para disminuir las posibles complicaciones.

En EE.UU. ya se ha lanzado la siguiente generación de este mismo implante, con avances en la prótesis y en la instrumentación, que esperamos permita obtener mejores resultados en el futuro.

\section{AGRADECIMIENTOS}

Agradecemos al Dr. José Antonio Pareja Esteban su inestimable ayuda en el estudio de los datos estadísticos obtenidos.

\section{CONFLICTOS DE INTERESES}

Los autores declaran no tener conflictos de intereses.

\section{BIBLIOGRAFÍA}

I. Ericka A. Lawler, M. a. Total wrist arthroplasty. Bull Hosp Jt Dis. 2006;64:98- 105.

2. Nydick JA, G. S. Clinical outcomes of total wrist arthroplasty. J Hand Surg Am. 20 I 2;37(8): I 580-4.
3. Ferreres A, Lluch A, del Valle M. Universal total wrist arthroplasty: midterm follow-up study. J Hand Surg. 201 1;36A:967-73.

4. Arnold-Peter C. Weiss, M. a. Total wrist replacement. Med Health R I. 20 I 2;95(4): I I7-9.

5. Rosales RS, Delgado EB, Diez de la Lastra I. Evaluation of the Spanish versión of the DASH and carpal tunnel syndrome health-related quality-oflife instruments: cross-cultural adaptation process and reliability. J Hand Surg Am. 2002;27:334-43.

6. Amit Gupta, M. F. Total wrist arthroplasty. Am J Orthop. 2008;37(8): I2-6.

7. Christi M. Cavaliere \& Adam J. Oppenheimer, K. C. Reconstructing the rheumatoid wrist: a utility analysis comparing total wrist fusion and total wrist arthroplasty from the perspectives of rheumatologists and hand surgeons. J Hand Surg Am. 2010; 5:9-18.

8. Christi M. Cavaliere, K. C. A cost utility analysis of non-operative management, total wrist arthroplasty, and total wrist fusion in rheumatoid arthritis. J Hand Surg Am. 201 0;35 (3):379-9l.

9. Christi M. Cavaliere, K. C. A systematic review of total wrist arthroplasty compared with total wrist arthrodesis for rheumatoid arthritis. Plast Reconstr Surg: 2008; I 22(3):813-25.

10. Matthew B. A. McCullough, B. D. Post-operative analysis of patients of the universal 2 total wrist implant system. J Appl Biomech. 20 I2;28:466-72.

I I. Palmer AK, W. F. Functional wrist motion: a biomechanical.J Hand Surg Am. 1985; I O( I):39-46.

12. Yngvar Krukhaug, S. A. Results of 189 wrist replacements. A report from the Norwegian Arthroplasty Register. Acta Orthop. 201 I;82(4):405-9.

13. Mattew C. Anderson, B. D. Total wrist arthroplasty. Hand Clin. 2005;21:621-30.

14. Adams, B. D. Total wrist arthroplasty. J Hand Surg Am. 200 I;1:236-48. 